\title{
Validación de un Cuestionario Autoadministrado para la Evaluación del Desarrollo Psicomotor
}

\author{
LUISA SCHONHAUT B. ${ }^{1}$, PATRICIA SALINAS A. ${ }^{1}$, IVÁN ARMIJO R. ${ }^{2}$, \\ MARIANNE SCHÖNSTEDT G. ${ }^{3}$, JORGE ÁLVAREZ L. ${ }^{1}$, MARCOS MANRÍQUEZ O. ${ }^{1}$ \\ 1. Facultad de Medicina Clínica Alemana, Universidad del Desarrollo. \\ 2. Psicólogo. Escuela Psicología, Universidad del Desarrollo. \\ 3. Terapeuta. Facultad de Medicina Clínica Alemana, Universidad del Desarrollo.
}

\begin{abstract}
Validation of a Parent Completed Developmental Screening Questionnaire

Introduction: Psychomotor Development (PMD) is an essential component of health care. Several authors have attempted to facilitate this evaluation through quick and inexpensive tools that do not prolong the time of consult. The "Ages and Stages Questionnaire" (ASQ) is a well-known and validated parentdirected survey. Objective: To evaluate the psychometric validity of ASQ, through comparison with Bayley's Scale of Infant Development (BSID), a universally accepted tool which measures similar parameters, and is administered by an expert. Patients and Methods: Children seen at an upper-middle class Private Clinic in Santiago, Chile were admitted to this study, with the exception of pre-term newborns, or children with known neurological, genetic or metabolic disease. Groups were formed at 8,18 and 30 months of age. A sample of nearly 40 children per age Group was formed. After parents responded to the ASQ, an expert, blind to the results of the ASQ, evaluated each patient within two weeks. Results: Total number of children enrolled was 119. PMD deficit was scored in $19.3 \%$ of children according to ASQ, $14.3 \%$ according to BSID (not significant). While the results obtained with both tests were found to be correlated $(\mathrm{r}=0.52)$, scores obtained through BSID were lower among children with deficit, compared to those rated through ASQ $(\mathrm{p}<0.001)$. The sensitivity of ASQ was $58.8 \%$ and the specificity was $87.2 \%$. Conclusions: Both tests are correlated. ASQ was found to be highly specific, suggesting its use as a screening tool in outpatient clinics, at least in a similar a population. These results should be reevaluated after national validation, to determine cutting points and test potency in all populations.

(Key words: Screening, psychomotor development, ages and stages questionnaires, pediatric outpatient clinic).

Rev Chil Pediatr 2009; 80 (6): 513-519
\end{abstract}

Trabajo recibido el 18 de agosto de 2009, devuelto para corregir el 21 de septiembre de 2009, segunda versión el 02 de octubre de 2009, aceptado para publicación el 17 de octubre de 2009.

Proyecto financiado por el concurso SOCHIPE 208013 en asociación con el proyecto FONIS SA7i20043.

Correspondencia a:

Dra. Luisa Schonhaut B.

E-mail: Ischonhaut@alemana.cl 


\section{RESUMEN}

Introducción: La evaluación del desarrollo psicomotor (DSM) es un componente esencial en la supervisión de salud. Distintos investigadores han buscado formas de facilitar esta evaluación en forma rápida y económica, sin prolongar el tiempo destinado a la consulta pediátrica. El Ages and Stages Questionnaires (ASQ) es un cuestionario de auto-reporte de los padres, validado ampliamente para este fin. Objetivo: Evaluar las propiedades psicométricas del ASQ. Para eso se compara con la Escala de Bayley III de Desarrollo Infantil (BSID), el cual es universalmente aceptado, mide parámetros similares, y es administrado por un experto. Pacientes y Métodos: Estudio transversal analítico. En forma secuencial se enrolaron niños de 8, 18 y 30 meses, de nivel socio económico medio alto, que acudieron a control sano en una Clínica privada de Santiago de Chile. Se excluyeron los nacidos de pretérmino o con enfermedad neurológica, genética o metabólica conocida. Se obtuvo una muestra cercana a 40 niños por grupo etáreo. Tras responder el ASQ, los niños fueron evaluados con la BSID III, por un experto, ciego a los resultados ASQ. La latencia entre ambas evaluaciones no superó las dos semanas. Resultados: Participaron 119 niños. La frecuencia global de déficit de DSM fue de 19,3\% de acuerdo al ASQ y 14,3\% según el BSID (no significativo). El rendimiento promedio del BSID fue inferior en el grupo de niños con déficit según el ASQ comparado con el grupo con ASQ normal $(\mathrm{p}<0,001)$. Se demostró correlación entre ambos test $(\mathrm{r} 0,52)$. La sensibilidad del ASQ fue 58,8\% y la especificadad fue 87,2\%. Conclusiones: Se demostró una adecuada correlación entre el ASQ y el BSID. El ASQ resultó altamente específico, por lo que podría ser usado como método de screening del DSM en la consulta pediátrica. Estos resultados deberían ser reevaluados luego de completar la validación nacional, para establecer los puntos de corte y real potencia del test.

(Palabras clave: Screening, desarrollo psicomotor, Ages and Stages Questionnaires).

Rev Chil Pediatr 2009; 80 (6): 513-519

\section{Introducción}

La evaluación desarrollo psicomotor (DSM) es considerada parte esencial de la supervisión de salud en pediatría.

Tanto en el ámbito nacional ${ }^{1}$, como a nivel internacional ${ }^{2}$, se recomienda vigilar la progresión del desarrollo en los controles de salud y la aplicación de pruebas estandarizadas de cribado frente a la sospecha clínica de déficit o bien a edades específicas. La Academia Americana de Pediatría recomienda el uso de test estandarizados a los 9, 18 y 30 meses de edad, y la norma técnica del Ministerio de Salud Nacional, a partir del año 2008, lo indica a $\operatorname{los} 8,18$ y 36 meses.

Se ha observado que la incorporación de estas pruebas en la práctica rutinaria, favorece la pesquisa precoz de las alteraciones del desarrollo y permite una intervención temprana y oportuna. También motiva la preocupación de los padres, promueve la estimulación en el hogar $^{3}$ y aumenta el grado de satisfacción con la consulta pediátrica, fortaleciendo la relación entre el médico, el paciente y su familia ${ }^{4}$.
No obstante lo anterior y, debido a limitaciones de tiempo, costo y disponibilidad de personal entrenado, la mayoría de los pediatras no utiliza estas pruebas estandarizadas y basan su detección sólo en capacidades clínicas. Estudios han demostrado que mediante el juicio clínico, como único instrumento, no se logra detectar más allá de la mitad de los niños con trastornos del $\mathrm{DSM}^{5}$ o con problemas de salud mental ${ }^{6}$ antes del ingreso a educación escolar.

Entre las pruebas actualmente disponibles, la Escala de Evaluación de Desarrollo de Bayley (BSID) ha sido utilizada con éxito en diversos ambientes clínicos, tiene una amplia aceptación y ha demostrado ser útil en la pesquisa precoz de aquellos niños que requerirán alguna intervención precoz. Sin embargo, la aplicabilidad clínica masiva del BSID se ha visto imposibilitada al ser una evaluación compleja, que requiere de personal altamente entrenado y tiene un alto costo. Ha quedado por tanto reservado su uso para aquellos niños con factores de riesgo conocidos o al ámbito de la investigación clínica.

Con el propósito suplir las dificultades refe- 
ridas para la aplicación masiva rutinaria de pruebas estandarizadas, e involucrar más a los padres en el cuidado de los niños, ${ }^{7}$ se han desarrollado cuestionarios de auto-reporte. Estos instrumentos han demostrado ser altamente confiables $^{8-11}$, independiente del nivel educacional y socioeconómico de los cuidadores ${ }^{12,13}$, y requieren poco tiempo, sin prolongar el tiempo destinado a la visita médica ${ }^{14-18}$. Uno de dichos test, el Ages \& Stages Questionnaires $(\mathrm{ASQ})^{19}$, ya ha sido validado para su uso en varios países ${ }^{20,21}$.

El objetivo del presente estudio fue evaluar la correlación entre ASQ y el BSID a los 8, 18 y 30 meses, y medir las propiedades psicométricas del ASQ en niños sanos, de nivel socioeconómico medio alto, en Santiago de Chile.

\section{Pacientes y Métodos}

Estudio de diseño transversal analítico. El Universo está conformado por lactantes y preescolares de término de nivel socioeconómico medio alto, que realizan en forma habitual su control de supervisión de salud en las consultas de pediatría ambulatoria de una Clínica Privada de Santiago de Chile, entre los meses de mayo 2008 y junio 2009.

La muestra fue de oportunidad, se reclutó en forma secuencial a todos los lactantes y preescolares entre 6 y 30 meses, hasta completar el tamaño muestral, que fue definido en 40 niños por grupo etáreo.

Se excluyeron los niños con diagnóstico conocido de enfermedad neurológica, congénita o metabólica, los nacidos prematuros (menores de 37 semanas de edad gestacional), aquellos cuyo adulto responsable no tuviese como idioma nativo el español y los que no completaron la evaluación con el BSID antes de 30 días desde que fue respondido el ASQ. Dado que la participación fue voluntaria, se excluyeron también todos aquellos casos en el que el adulto responsable declinó participar. Cada niño se incluyó sólo una vez en el estudio.

A los cuidadores principales de los niños enrolados, se les solicitó firmar el consentimiento informado y completar un formulario con datos de identificación, información demográfica y de riesgo biológico. Posteriormente se les solicitó llenar el ASQ y fijar una cita, dentro del menor plazo posible, idealmente no superior a 1 ó 2 semanas, para la aplicación del BSID por una Terapeuta Ocupacional acreditada para dicha evaluación y ciega a los resultados del ASQ.

El proyecto fue aprobado por el Comité de Ética de la Clínica Alemana, Universidad del Desarrollo.

\section{Instrumentos}

Ages and Stages Questionnaire (ASQ) ${ }^{22}$ : Cuestionario escrito de autoreporte para padres que chequea el nivel de DSM para niños desde el nacimiento hasta los 5 años de edad. Se encuentra traducido a varios idiomas. A pesar de existir una versión estandarizada en español, que ha sido usada en otros centros en el extranjero, se realizó una adaptación lingüística para nuestro país previo al inicio del estudio.

Se dispone de cuestionarios para 19 etapas en este rango de edad. Consta de tres secciones: i) preguntas demográficas, ii) 30 preguntas enfocadas a cinco áreas del desarrollo (comunicación, motor grueso, motor fino, solución de problemas y personal/social) y iii) siete preguntas libres orientadas a recoger la preocupación de los padres.

De acuerdo a las recomendaciones del manual del usuario, se consideró rendimiento deficitario si el niño evaluado tenía al menos en un dominio un puntaje $<$ a -2DS del promedio.

Escala de Bayley III de Desarrollo Infantil ( $3^{\mathrm{a}}$ edición 2005) ${ }^{23}$ : Escala que evalúa el desarrollo en niños de 1 a 42 meses, mediante tres escalas diferenciadas. De acuerdo al manual, se consideró rendimiento deficitario si el niño evaluado tenía al menos en un dominio un puntaje bajo-1DS del promedio.

\section{Análisis estadístico}

Se realizó un análisis descriptivo de puntajes segmentados por edad de la muestra, con el fin de detectar el comportamiento típico en cada subgrupo. Se midió la concordancia de Person entre las evaluaciones de ASQ y el BSID y se analizaron las propiedades psicométricas del ASQ. 


\section{Resultados}

Se aplicaron los test de ASQ y BSID a 119 niños que cumplían los criterios de inclusión. La totalidad de ellos tenía al menos uno de los padres con educación superior y provenían de familias de nivel socio económico medio alto.

Veintitrés niños (19,3\%) fueron catalogados con déficit de acuerdo al criterio del ASQ y $14,3 \%$ de acuerdo al BSID, diferencia no significativa. Según ambas evaluaciones la prevalencia de déficit fue mayor en los niños de género masculino, siendo significativo sólo en el análisis del ASQ $(\mathrm{p}=0,04)$.

Los lactantes de 8 meses mostraron la frecuencia más alta de déficit del DSM, que alcanzó al 30\% en ambas evaluaciones. A los 18

Tabla 1. Frecuencia déficit del desarrollo psicomotor por grupo etáreo, acorde a ambas pruebas

\begin{tabular}{lcrrr}
\hline & Muestra & \multicolumn{1}{c}{ ASQ* } & \multicolumn{2}{c}{ BSID** } \\
\hline 8 meses & 43 & $13(30,2 \%)$ & $13(30,2 \%)$ \\
18 meses & 39 & $6(15,4 \%)$ & 3 & $(7,7 \%)$ \\
30 meses & 37 & $4(10,8 \%)$ & 1 & $(2,7 \%)$ \\
Total & 119 & $23(19,3 \%)$ & $17(14,28 \%)$
\end{tabular}

*Ages and Stages: Diferencia en frecuencia de déficit por edad no significativo $p=0,072$.

**Escala de Bayley de Desarrollo Infantil: Diferencia en frecuencia de déficit por edad significativa $p=0,001$.

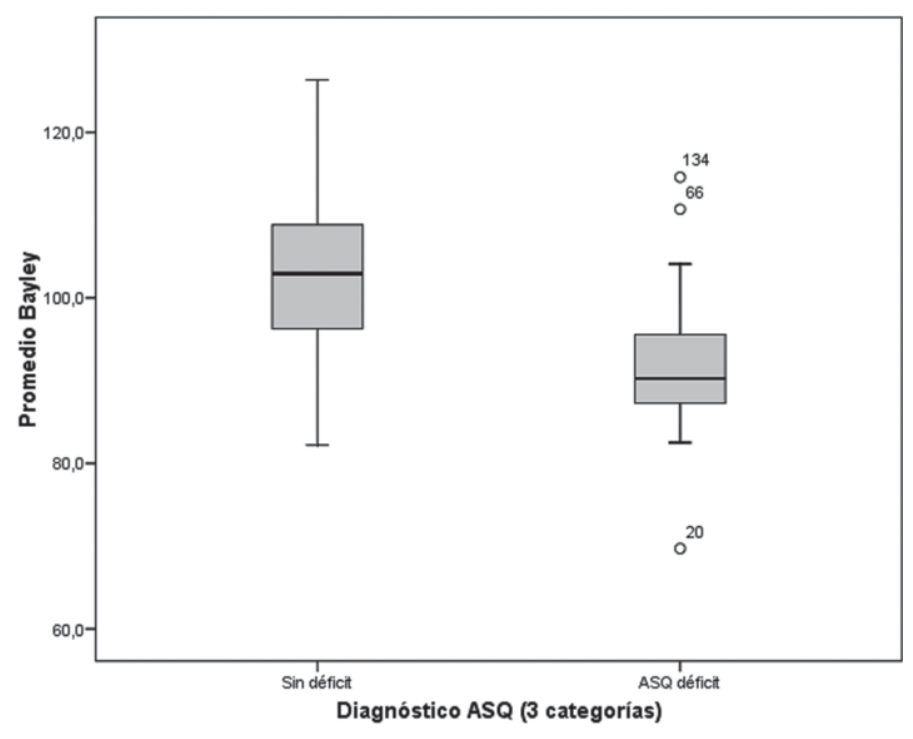

Figura 1. Puntaje promedio en Escala de Bayley del Desarrollo Infantil en niños con déficit del desarrollo psicomotor acorde al ASQ comparado con niños sin déficit. 


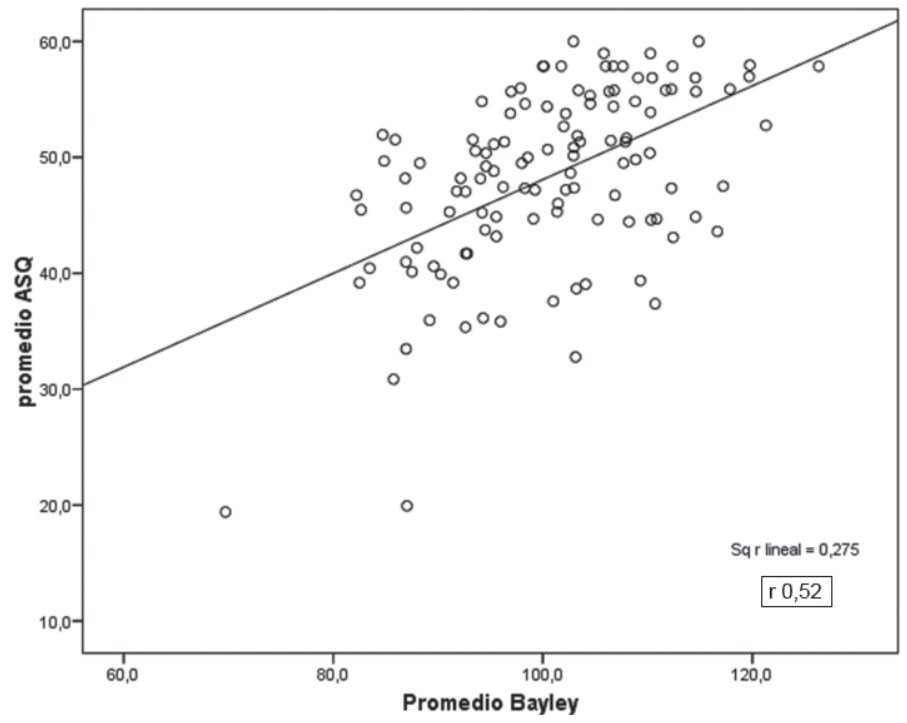

Figura 2. Correlación global entre los promedios de ambos test.
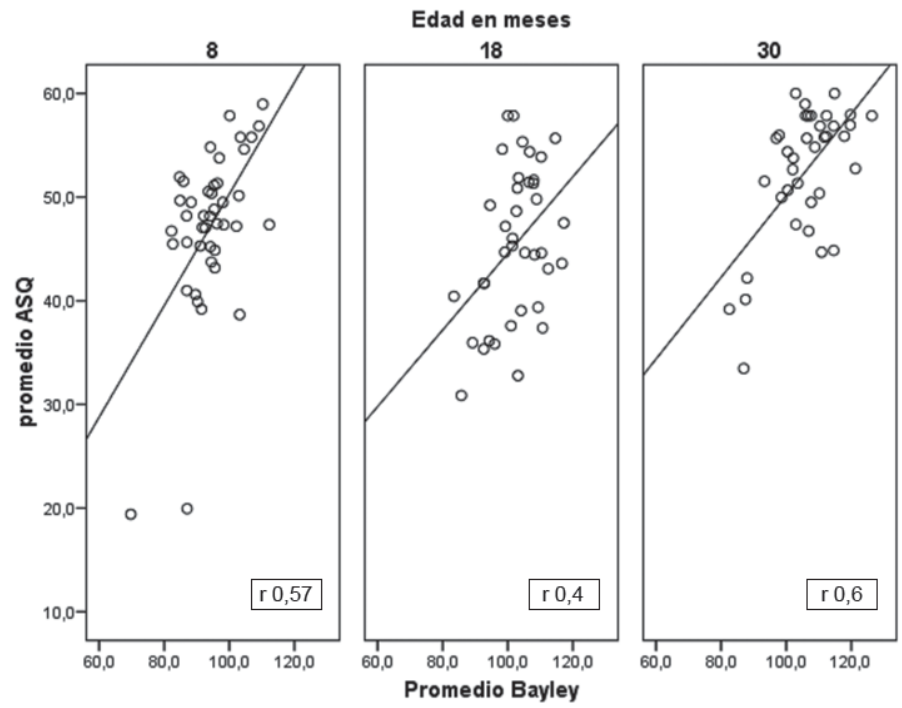

Figura 3. Correlación entre ambos test por grupo etáreo.

\section{Discusión}

El presente estudio forma parte de un proyecto de validación del ASQ en nuestro medio ${ }^{24}$. El propósito es contar con una herramienta de screening del DSM que pueda ser empleada en un programa nacional de promoción de la salud infantil, tanto en el ámbito público como en la consulta pediátrica del sistema privado de atención.

Pudimos constatar un gran interés y excelente acepta- ción por parte de los padres participantes en el estudio. Es importante tener presente que nuestro estudio representa un segmento de la población chilena, ya que prácticamente la totalidad de los padres tenía estudios superiores y pertenecía al estrato socio económico medio alto a alto. Estudios internacionales revelan una relación entre la proporción de respuesta de los cuestionarios, cifra que podría ser interpretada como un reflejo del interés y prioridad asignada al desarrollo de los niños, y el nivel educacional de los padres ${ }^{21,25}$.

La prevalencia encontrada de déficit del DSM fue de 19,3\% según el ASQ y 14,3\% de acuerdo al BSID, lo cual concuerda con a la reportada por en la Encuesta de Calidad de vida y Salud para niños provenientes del sector socio económico medio alto del país ${ }^{26}$.

La frecuencia de déficit del DSM disminuyó con la edad, lo que podría relacionarse con el efecto protector del medio ambiente en que se desarrollan los niños participantes en el estudio, en contraste con lo que ocurre con niños provenientes de familias que viven en condición de pobreza $^{27}$. La descripción del desarrollo de los niños participantes del estudio se detalla in extenso en una próxima publicación ${ }^{28}$.

Encontramos buena correlación entre los test de ASQ y el BSID. En cuanto a las propiedades psicométricas del ASQ, como test de screening, la alta especificidad y valor predictivo negativo implican que, si el ASQ está normal, es muy poco probable que el niño tenga un déficit en su desarrollo, es decir, minimiza la posibilidad de no detectar a niños con un verdadero retraso en el desarrollo, lo que corresponde a una de las principales fortalezas del cuestionario $^{29}$. 
Por el contario, los valores obtenidos de sensibilidad y valor predictivo positivo reflejan que sólo la mitad de los niños que caen bajo la línea de corte del ASQ tendrían un problema real del DSM. Al respecto hay que considerar que el grupo de niños con déficit según el ASQ tuvo un rendimiento significativamente inferior en el BSID. Los test de auto-reporte se basan en la comparación que hacen los padres con otros niños de la edad y, pese a tener un rendimiento normal, podrían ser estos niños de mayor cuidado, limítrofes en su desarrollo psicomotor o social y que requerirán servicios de apoyo posterior ${ }^{30}$.

Pese a que estos hallazgos debieran ser reevaluados luego de completar la validación nacional, para establecer los puntos de corte y real potencia del test, los resultados obtenidos nos invitan a implementar el ASQ como método estandarizado de apoyo en la evaluación del desarrollo infantil en la consulta pediátrica ambulatoria. Pensamos que esto indudablemente se relacionaría con mejora en la calidad de atención y mayor satisfacción tanto de los pacientes como de los profesionales.

\section{Conclusiones}

Se demostró una adecuada correlación entre el ASQ y el BSID. A pesar que la sensibilidad encontrada para el ASQ en nuestro estudio no fue tan alta, es comparable con estudios internacionales. La elevada especificidad y valor predictivo negativo reflejan la capacidad del cuestionario de identificar correctamente a los niños verdaderamente normales, debiendo derivar a una evaluación más completa en aquellos niños con rendimiento deficitario. Si bien falta completar la validación nacional, estos hallazgos, sumados a otras ventajas reportadas en la literatura, como la oportunidad de mejorar la alerta del equipo de salud y el mayor involucramiento y satisfacción de los padres, además del bajo costo y fácil aplicabilidad, hace altamente recomendable la implementación rutinaria del ASQ en la consulta pediátrica, para la evaluación estandarizada del DSM en niños de 8,18 y 30 meses.

\section{Referencias}

1.- Ministerio de Salud: Normas técnicas de evaluación y estimulación del desarrollo psicomotor en el niño y la niña menor de 6 años. Santiago Chile 2004. [accedido 26 de mayo 2007] disponible en http://www.minsal.cl/ ici/S_1/salud_nino/Manual.pdf

2.- Council on Children with Disabilities, Section On Developmental Behavioral Pediatrics, Bright Futures Steering Committee and Medical Home Initiatives For Children With Special Needs Project Advisory Committee: Identifying Infants and young children with developmental disorders in the Medical Home: An Algorithm for Developmental Surveillance and Screening. Pediatrics 2006; 118: 405-20.

3.- Marks K: Should General Pediatricians Not Select the Ages \& Stages Questionnaire in Light of the Rydz et al Study. Pediatrics 2007; 120: 457-8.

4.- Halfon $N$, Regalado M, Sareen H, Inkelas M, et al: Assessing Development in the Pediatric Office. Pediatrics 2004; 113 (6): 1926-33. http://pediatrics. aappublications.org/cgi/content/full/113/6/S1/1926? maxtoshow $=\&$ HITS $=10 \&$ hits $=10 \&$ RESULTFORMAT $=$ \&fulltext $=$ HALFON\&andorexactfulltext $=$ and\&searchid $=$ 1\&FIRSTINDEX $=0 \&$ sortspec $=$ relevance $\&$ resource type $=$ HWCIT

5.- Palfrey J S, Singer J D, Walker D K, Butler J A: Early identification of children's special needs: a study in five metropolitan communities. J Pediatr 1987; 111: 651-65.

6.- Lavigne J, Binns H, Kaufer Christoffel K, Rosenbaum D: Behavioral and Emotional Problems among Preschool Children in Pediatric Primary Care: Prevalence and Pediatricians' Recognition. Pediatrics 1993; 91 (3): 649-55.

7.- Schonhaut L, Álvarez J, Salinas P: El pediatra y la evaluación del Desarrollo Psicomotor. Rev Chil Pediatr 2008; 79 supl (1): 26-31.

8.- Bailey D, Hebbeler K, Scarborough A, Spiker D, Mallik $S$ : First Experiences With Early Intervention: A National Perspective. Pediatrics 2004; 113 (4): 88796.

9.- ASQ, Technical report, (accedido el 14 de agosto de 2007). Descargado desde http://www.brookespublishing. com/store/books/bricker-asq/index.htm

10.- Regalado M, Halfon $N$ : Primary Care Services promoting optimal child development from birth to age 3 years. Arch Pediatr Adolesc Med; 155: 1311-22.

11.- Yu LM, Hey E, Doyle LW, Farrell B, Spark P, Altman DG, Duley L; Magpie Trial Follow-Up Study 
Collaborative Group: Evaluation of the Ages and Stages Questionnaires in identifying children with neurosensory disability in the Magpie Trial follow-up study. Acta Paediatr 2007; 96 (12): 1803-8.

12.- Glascoe FP, Dworkin PH: The role of parents in the detection of developmental and behavioral problems. Pediatrics 1995; 95: 829-36.

13.- Squires J, Potter L, Bricker D, Lamorey S: Parentcompleted developmental Questionnaires: Effectiveness with low and middle income parents. Early Childhood Research Quarterly 1998; 13 (2): 345-54.

14.- Dobrez D, Sasso AL, Holl J, Shalowitz M, León S, Budetti P: Estimating the cost of developmental and behavioral screening of preschool children in general pediatric practice. Pediatrics 2001; 108: 913-22.

15.- Sices L, Drotar D, Keilman A, H. Kirchner L, Roberts $D$, Stancin T: Communication About Child Development During Well-Child Visits: Impact of Parents' Evaluation of Developmental Status Screener With or Without an Informational Video Pediatrics 2008; 122: e1091-9.

16.- Rydz D, Srour M, Oskoui M, et al: Screening for Developmental Delay in the Setting of a Community Pediatric Clinic: A Prospective Assessment of ParentReport Questionnaires. Pediatrics 2006; 118: 117886.

17.- Hix-Small H, Marks K, Squires J, Nickel R: Impact of Implementing Developmental Screening at 12 and 24 Months in a Pediatric Practice. Pediatrics 2007; 12082: 381-9.

18.- Schonwald A, Horan K, Huntington N: Developmental Screening: Is There Enough Time? Clinical Pediatrics 2009; 48 (6): 648-55.

19.- Squires J, Bricker D, Potter LW: Revision of a ParentCompleted Developmental Screening Tool: Ages and Stages Questionnaires. Journal of Pediatric Psychology 1997; 22 (3): 313-28.

20.- Eun Young Kim, In Kyung Sung: The Ages and Stages Questionnaires: screening for developmental delay in the setting of a pediatric outpatient clinic. Korean
Journal of Pediatrics 2007; 50 (11): 1061-6.

21.- Richter J, Harald J: A validation study of the Norwegian version of the Ages and Stages Questionnaires. Acta Paediatrica 2007; 96: 748-52.

22.- Squires J, Bricker D, Potter L: Ages and Stages Questionnaires: User's Guide. 2nd ed. Baltimore, MD: Paul Brookes Publishing; 1999.

23.- Bayley N: Bayley Scales of Infant and Toddler Development. Socio-Emotional Scale.Third Edition. PsychCorp. 2005.

24.- Armijo I, Cordero M: Adaptación, validación y estandarización de un instrumento de auto-reporte para padres en la detección de rezagos del desarrollo de niños y niñas entre 6 y 18 meses de edad. Proyecto FONIS SA7i20043.

25.- Klamer A, Lando A, Pinborg A, Greisen G: Ages and Stages Questionnaire used to measure cognitive deficit in children born extremely preterm. Acta Paediatrica 2005; 94: 1327-9.

26.- Subsecretaría de Salud Pública División de Planificación Sanitaria: II Encuesta de Calidad de Vida y Salud, Chile 2006. Disponible en: http://epi.minsal.cl/ epi/html/sdesalud/calidaddevida2006/Informe $\% 20$ Final\%20Encuesta $\% 20 \mathrm{de} \% 20$ Calidad $\% 20 \mathrm{de} \% 20$ Vida $\% 2$ 0y\%20Salud\%202006.pdf

27.- Schonhaut L, Maggiolo M, Herrera M, Acevedo K Y, García M: Lenguaje e inteligencia de preescolares: análisis de su relación y factores asociados. Rev Chil Pediatr 2008; 79 (6): 600-6.

28.- Schonhaut L, Schonstedt M, Álvarez J, Salinas P, Armijo I: Evolución del desarrollo psicomotor en niños de nivel socioeconómico alto. Revista Chil Pediatr. En prensa.

29.- Skellern C, Roger Y, O'Callaghan M: A parentcompleted developmental questionnaires: Follow up of ex-premature infants. Journal of Paediatrics \& Child Health 2001; 37 (2): 125-9.

30.- Glascoe F: Parents concerns about children development, prescreening technique or screening test? Pediatrics 1997; 99 (4): 522-8. 